\title{
VP-RDF: An RDF Based Framework to Introduce the Viewpoint in the Description of Resources
}

\author{
Ouahiba Djama* \\ Lire Laboratory, University of Abdelhamid Mehri Constantine 2, Ali Mendjeli, Constantine, Algeria
}

\begin{abstract}
The description of resources and their relationships is an essential task on the web. Generally, the web users do not share the same interests and viewpoints. Each user wants that the web provides data and information according to their interests and specialty. The existing query languages, which allow querying data on the web, cannot take into consideration the viewpoint of the user. We propose introducing the viewpoint in the description of the resources. The Resource Description Framework (RDF) represents a common framework to share data and describe resources. In this study, we aim at introducing the notion of the viewpoint in the RDF. Therefore, we propose a View-Point Resource Description Framework (VP-RDF) as an extension of RDF by adding new elements. The existing query languages (e.g., SPARQL) can query the VP-RDF graphs and provide the user with data and information according to their interests and specialty. Therefore, VP-RDF can be useful in intelligent systems on the web.
\end{abstract}

Keywords - Resource Description Framework (RDF), SPARQL, web resource, viewpoint.

\section{INTRODUCTION}

A web resource is anything, which we can be found on the World Wide Web [1]. It can include text documents, HTML pages [2], e-mails, videos, traditional databases, flat files, knowledge bases, topical content and programs [3].

A web resource appears in many contexts (e.g., semantic web, linked data and internet of things). The Semantic Web [4] allows treating the semantics in the web resource. It provides some technologies (e.g., Resource Description Framework (RDF)), which allow describing the web resources and their semantic properties [5], [6].

The vision of the Semantic Web is to extend the principles of the Web from documents to data [7]. Then, linked data [8], [9] has emerged.

Linked data allow publishing structured data that can be connected together. This also necessitates the exploitation of a common framework (e.g., RDF) [7]. This allows data to be shared [7]. RDF allows defining and describing the relations among data (i.e., resources) in linked data.

Polleres et al. in [10] have indicated that, "Linked Data promises that a large portion of Web Data will be usable as one big interlinked RDF database". There are several tools to query data, which are described through such relationships in the RDF model. SPARQL [11] is one of these tools.
Generally, the web users do not share the same interests and the same viewpoints about resources and the relationships between them. Therefore, each user wants that the web provides data and information according to their interests and specialty. However, the web cannot provide the user with data and information according to their interests and specialty because the existing query languages cannot take into consideration the viewpoint in the data querying on the web. For example, in the real estate site, a user wants to know all the existing properties of an apartment that describe its size without citing these properties (e.g., height, area, the number of rooms, etc.). The user is interested only in information about the size. The existing tools provide user with all properties of this apartment without filtering (e.g., height, area, the number of rooms, price, rent, location, etc.). Then, the user will select responses that belong to their interests.

The introduction of the viewpoint in the data querying does not give results. We think that it is preferable to introduce the viewpoint in the description of the resources.

In this paper, we are interested in the problem of the integration of the viewpoint in the description of the web resources (data) and their relationships in the context of semantic web, linked data and even the internet of things.

RDF represents a foundation technology for semantic web and linked data. Therefore, we aim at introducing the notion of the viewpoint in RDF. We will propose VP-RDF (View-Point Resource Description Framework) as an extension of RDF by adding new elements. VP-RDF allows introducing the viewpoint in the description of the resources (data) and their relationships.

In the following section, we present a state of art on the notion of the viewpoint. Then, in Section 3, we explain the framework of VP-RDF that we propose. After that, in Section 4 we apply the proposed framework to some use cases. Section 5 presents the document querying that is constructed with VPRDF. Section 6 provides some results of the research and some areas for future work.

\footnotetext{
*Corresponding author's e-mail: djama_ouahiba@umc.edu.dz 


\section{STATE OF THE ART}

Different definitions of the notion of the viewpoint have been proposed by different authors.

Some of them assume that the viewpoint corresponds to the perception of an object according to the observer's position (e.g., the work in [12]). In this case, Djama and Boufaida [2] have given an example of symbol ' 9 ': the observer, who is in the up position, sees number 6 and who is in the down position, sees number 9 .

Some other authors consider the viewpoint as a partial definition of an object (e.g., the work in [2], [13]-[17]). This partial definition describes only some set of properties of this object.

For example, in the real estate domain [2], the object apartment is defined according to viewpoint Finance by the properties: rent, price, etc. These properties represent a subset of all the properties of the object apartment. Therefore, these properties represent a partial definition of the object apartment. The constraints on the value of the property price allow creating two concepts: expensive apartment and cheap apartment. These two concepts are linked to the viewpoint Finance.

Another example, in the real estate domain also, the object tenant is defined according to viewpoint Finance by the properties: salary, etc. Therefore, we can obtain the concept rich tenant that is linked to the viewpoint Finance.

In the assertion "rich tenant lives in expensive apartment", the relation lives will be defined according to viewpoint Finance.

Before presenting the related work, we explain the difference between Viewpoint, Context and Opinion in the following subsection.

\section{A. Difference Between Viewpoint, Context and Opinion}

There are two other notions that are near the notion of the viewpoint: "opinion" and "context".

The opinion is a judgment based on non-rational arguments that are related to feelings [18]. For example, "For me, England is the most beautiful country". However, the viewpoint is a judgment based on rational arguments.

For example, viewpoint 1: "This apartment is an expensive apartment because its price is very height". Viewpoint 2: "It is a large apartment because its area is very large". The two viewpoints speak on the same apartment. Viewpoint 1 is interested in the finance. However, Viewpoint 2 is interested in the size.

The context is a judgment based on rational arguments that represent a set of conditions [19]-[24].

For example, there are two types of geometry: Euclidean geometry (the geometry of 2D) and solid geometry (the geometry of 3D or Euclidean space). Euclidean geometry and solid geometry represent two contexts. The condition of Euclidean geometry: the objects are defined by only two dimensions. However, in solid geometry, there are other conditions.

In the context of Euclidean geometry, parallel lines cannot intersect. However, they can intersect in the context of solid geometry.

\section{B. Related Work}

Several works have been realised on the notion of the viewpoint. Some of them are interested in the integration of the viewpoint in the ontology (e.g., [14], [15], [25]-[32]).

Hemam et al. [33] have developed a method that allows coupling the notion of the viewpoint and the notion of the composite concepts in the ontology. Then, Hemam in [34] has introduced the probabilistic reasoning in this method.

Djezzar and Boufaida [35] are interested in the classification of an individual in an ontology. This ontology allows taking into consideration the viewpoint to represent knowledge.

Djakhdjakha et al. [36] have developed a method that aligns ontologies, which allow representing the notion of the viewpoint.

Djama and Boufaida [2] have developed an approach that allows acquiring the instances of concepts from a text resource. These concepts are defined in the ontology that allows representing the viewpoint (the multi-viewpoint ontology).

Djama and Boufaida [37], [38] have proposed an approach that allows using multi-viewpoints ontology to annotate an XML document.

All these works are interested in the representation of the viewpoint in the ontology. However, ontology is a kind of dictionary for the web. It is not always obligatory to refer to an ontology to describe a resource on the web.

Sometimes, it suffices to use a simple framework (e.g., RDF) to describe a resource. However, there is no work in the literature that allows introducing the notion of the viewpoint in RDF.

\section{REPRESENTATION OF THE PROPOSED FRAMEWORK}

VP-RDF is an extension of the RDF, where we propose adding new elements. These elements are:

(1) Viewpoint: a specific resource, where the set of viewpoints represent subset of RDF resources.

(2) Predicate with_Viewpoint: a specific predicate that expresses a relationship between the subject (an RDF resource) and a viewpoint.

(3) VP-RDF triplet (Subject, Predicate, Object). The Subject is an RDF resource that is different from Viewpoint. The Predicate is instance of Predicate_with_Viewpoint. The Object is an instance of Viewpoint.

Therefore, VP-RDF is based on the following foundations:

(1) Viewpoint;

(2) Predicate_with_Viewpoint;

(3) The principle of VP-RDF triplets;

(4) URI (Uniform resource identifier);

(5) The principle of the classical RDF triplets (Subject, Predicate, Object). The Subject and the Object are RDF resources that are different from Viewpoint. The Predicate is different from instances of Predicate_with_Viewpoint;

(6) The principle of the graphs.

Thus, VP-RDF is based on the idea of making two types of statements: the classical RDF statement (Subject, Predicate, Object) and the new type of statement that we propose. The latter is composed of (Subject, Predicate_with_Viewpoint, Viewpoint). 
In the following subsections, we present the formalization of the VP-RDF triplets, the vocabulary of the VP-RDF framework and the implementation of the VP-RDF elements.

\section{A. Formalization of the VP-RDF Triplets}

According to Patel-Schneider [39], the semantics of an RDF triplet can be translated into a first-order logic formula by using conjunctive, existential quantification and binary predicate.

\section{$\exists$ Subject, $\exists$ Object $:$ predicate (Subject, Object)}

Likewise, we can translate a VP-RDF triplet into a first-order logic formula as follows:

\section{$\exists$ Subject, $\exists$ Viewpoint :}

\section{Predicate_with_Viewpoint (Subject,Viewpoint)}

\section{B. Vocabulary of the $V P-R D F$}

VP-RDF supports the same set of RDF/RDFS classes and properties (e.g., rdf:List, rdfs:Resource, rdfs:Literal, rdf:type, rdfs:domain, etc.) plus new classes and properties that we propose to represent the viewpoint.

The proposed classes (Table I) are:

(1) VPrdf:Viewpoint: a proposed class that allows defining the set of the viewpoints.

(2) VPrdf:Predicate_with_Viewpoint: a proposed class that allows defining the property that links a resource to the viewpoint.

(3) VPrdf:Statement: the proposed class that allows defining the VP-RDF triplet.

The proposed properties are:

(1) VPrdf:Subject_Statement,

(2) VPrdf:Predicate_Statement, and

(3) VPrdf:Object_Statement.

These properties (Table II) allow defining the components of VPrdf:Statement, where:

- VPrdf:Predicate Statement is an instance of VPrdf:Predicate_with_Viewpoint;

- VPrdf:Object_Statement is an instance of VPrdf:Viewpoint.

TABLE I

VP-RDF CLASSES

\begin{tabular}{|l|c|c|}
\hline Class & Description & Definition by the RDF vocabulary \\
\hline VPrdf:Viewpoint & The class of Viewpoints & Subclass of "rdfs:Resource" \\
\hline VPrdf:Predicate_with_Viewpoint & The class of the property "Predicate_with_Viewpoint" & Subclass of "rdf:Property" \\
\hline VPrdf:Statement & The class of the VPrdf statements & Subclass of "rdf:Statement" \\
\hline
\end{tabular}

TABLE II

VP-RDF PROPERTIES

\begin{tabular}{|l|l|c|}
\hline Property & Domain & Range \\
\hline VPrdf:Subject_Statement & VPrdf:Statement & rdfs:Resources \\
\hline VPrdf:Predicate_Statement & VPrdf:Statement & VPrdf:Predicate_with_Viewpoint \\
\hline VPrdf:Object_Statement & VPrdf:Statement & VPrdf:Viewpoint \\
\hline
\end{tabular}

\section{Implementation of the VP-RDF Elements}

The VP-RDF elements are defined as subclasses of RDFS/RDF elements. The VP-RDF elements will be implemented in RDFS language. In this section, we show an example of the implementation of a VP-RDF class and an example of the implementation of a VP-RDF property.

Below, we show the implementation of VPrdf:Predicate_with_Viewpoint as a subclass of rdf:Property in RDFS language.

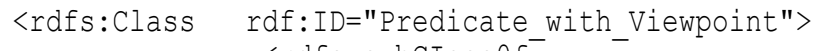

$\langle/$ rdfs:Class $\rangle$
Below, we show the implementation, in RDFS language, of the property VPrdf:Object_Statement as RDF Property that has domain VPrdf:Statement and range VPrdf:Viewpoint.

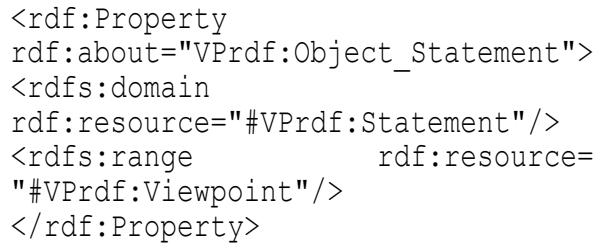

VPrdf:Statement and VPrdf:Viewpoint are implemented as subclasses of rdf:Statement and rdfs:Resource.

\section{Syntax of the VP-RDF Document}

We exploit the VP-RDF elements and the RDF elements to create a VP-RDF document. Gryaznov and Rusakov [40] have 
assumed that the apparent advantage of XML syntaxes is compatibility with existing XML tools and technologies (parsers, editors, XSLT, XQuery and XPath). We adopt the XML syntaxes to describe the structure of the VP-RDF document. The latter is a list of descriptions of resources by taking into the consideration the viewpoints. Each representation is announced via the $<$ VPrdf: Viewpoint Description $>$ tag.

To represent the VP-RDF elements, we propose the name space "VPrdf". Each < VPrdf: Viewpoint_Description> is defined by either the VP-RDF statements or RDF statements. The basic VP-RDF XML serialization syntax is represented as follows:

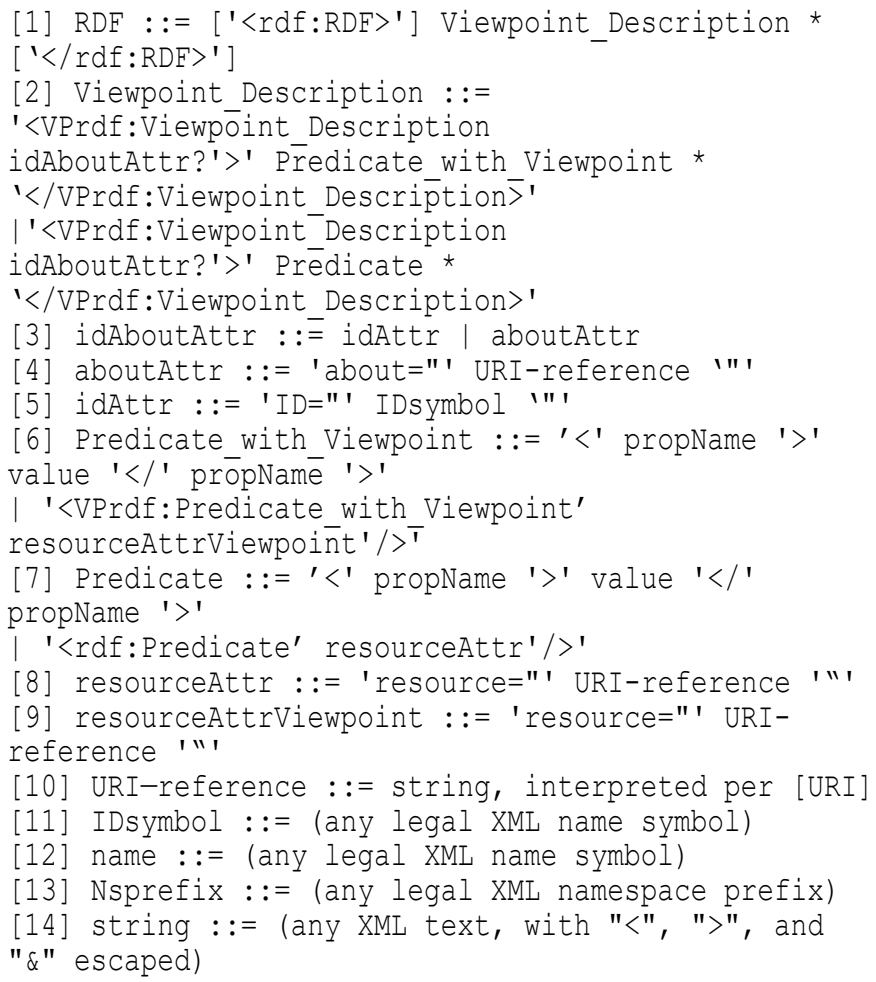

\section{USE CASES}

In this section, we present the application of our approach to some examples (as demonstrations). These examples represent all the existing simple cases of the description resources by taking into consideration the viewpoints. Then, the other cases are the combination between these cases.

There are five principal cases to link the description of resources to the viewpoint:

\section{A. Case 1: Resource Linked to a Given Viewpoint}

Example 1: In the real estate domain, the resource "Large_Apartment" is defined in the Size viewpoint. This case will be represented as follows.

\section{$(<$ Large_Apartment $>,<$ defined_in $>,<$ Size $>)$}

This case represents a relation between a resource and a viewpoint. It is represented by a VP-RDF triplet (statement).

\section{B. Case 2: A Resource Linked to Several Viewpoints}

Example: in the real estate domain, the resource "Tenant" is defined according to the Size viewpoint and Finance viewpoint. This case will be represented by a graph composed of two triplets. The first one is represented as follows:

$(<$ Tenant $>,<$ defined_according_to $>,<$ Size $>)$

The second one is represented as follows:

$(<$ Tenant $>,<$ belong_to $>,<$ Finance $>)$

In this case, the subject of the first triplet represents the subject of the second triplet. This example will be represented in VP-RDF (see Fig. 1).

This case is represented by a graph that is composed of two VP-RDF triplets.

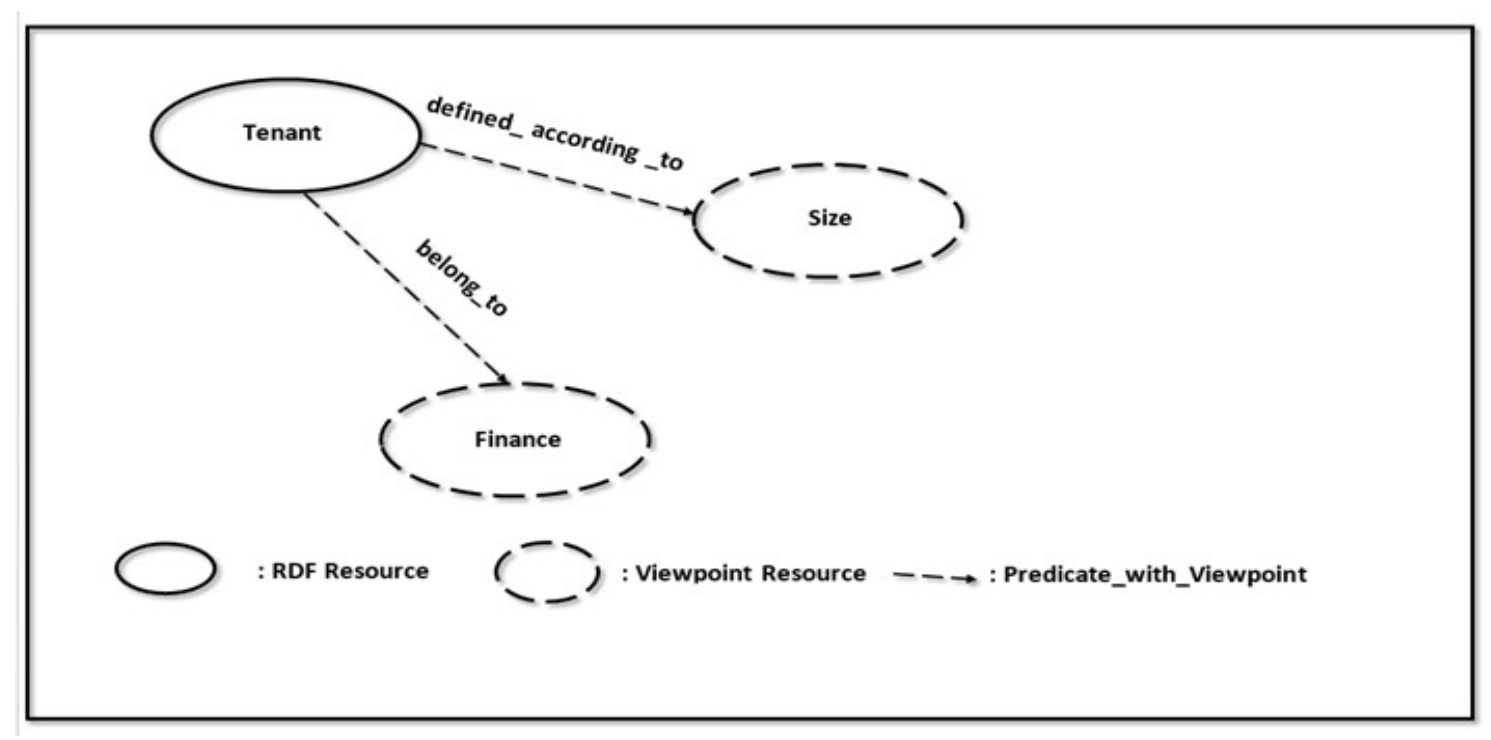

Fig. 1. RDF graph representation of an example of Case 2: a resource linked to several viewpoints. 
C. Case 3: A Resource Has a Property with a Resource. The Latter Is Linked to a Viewpoint

Example: In the education domain, the resource "Jacque" is described by the resource "Associate Professor". The resource "Associate_Professor" is linked to the viewpoint "University_Education". This case will be represented by a graph composed of two triplets. The first one is represented as follows:

$(<$ Jacque $>,<$ is $>,<$ Associate_Professor $>$ )
The second one is represented as follows:

$$
(<\text { Associate_Professor }>, \quad<\text { defined__according _to }>\text {, }
$$$$
<\text { University_Education }>\text { ) }
$$

In this case, the object of the first triplet represents the subject of the second triplet. This example will be represented in VPRDF (see Fig. 2).

This case is represented by a graph that is composed of an RDF triplet and a VP-RDF triplet.

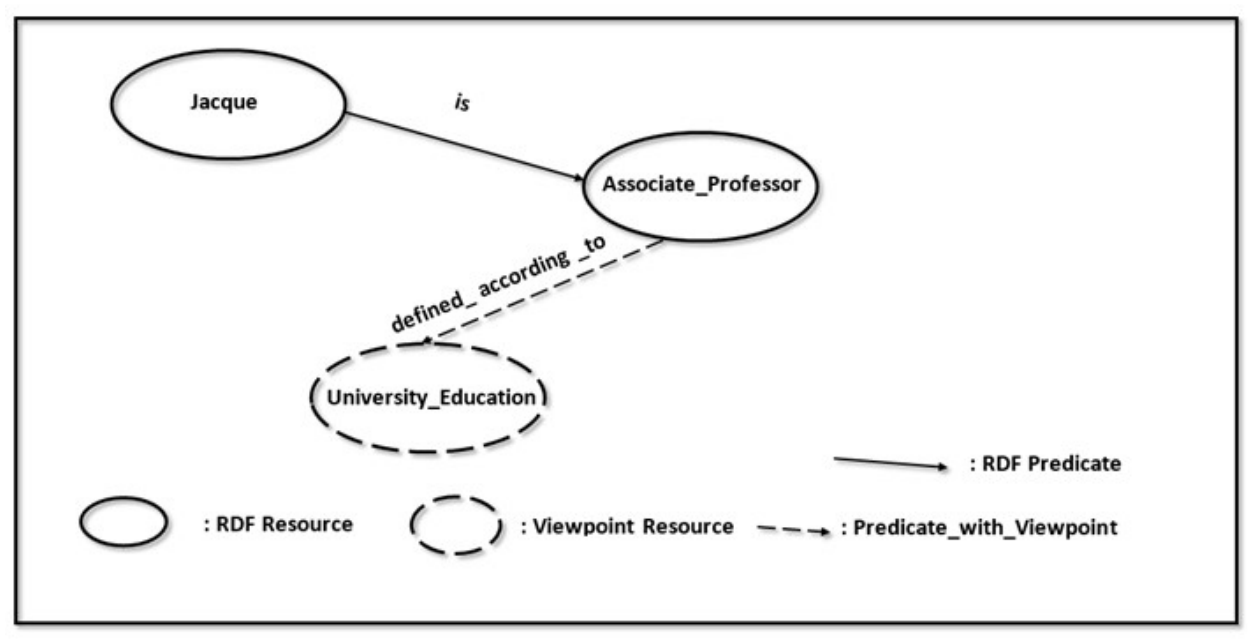

Fig. 2. RDF graph representation of an example of Case 3: a resource has a property with a resource. The latter is linked to a viewpoint.

D. Case 4: A Resource, Which Is Linked to a Given Viewpoint, Has a Property with Another Resource. The Latter Is Linked to Another Viewpoint

Example: In the real estate domain, the resource "Rich_Tenant" that appears in the Finance viewpoint lives_in a "Large_Apartment". The latter is defined in the Size viewpoint.

This case is represented in Fig. 3.
This case is represented by a graph that is composed of an RDF triplet and two VP-RDF triplets, where the subject of the RDF triplet becomes the subject of the first VP-RDF triplet and the object of the RDF triplet becomes the subject of the second VP-RDF triplet.

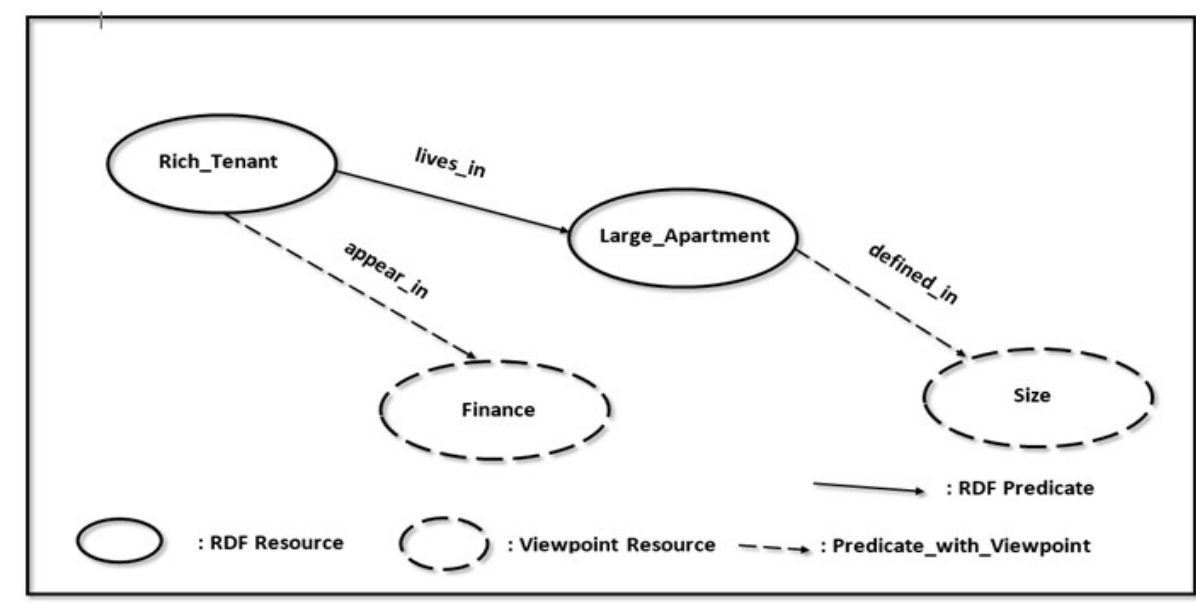

Fig. 3. RDF graph representation of an example of Case 4: a resource, which is linked to a given viewpoint, has a property with another resource. The latter is linked to another viewpoint. 


\section{E. Case 5: A Resource That Is Described by Another Resource.} This Description Is Defined According to a Given Viewpoint

Example: In the real estate domain, the resource "Johan" lives_in a "Large_Apartment" under the Size viewpoint.

In this case, the predicate "lives_in" is defined according to the Size viewpoint. To represent this case, first, it is necessary to create a class to represent the predicate "lives_in". Case 5 is demonstrated in Fig. 4.

This case represents a relation between an RDF predicate and a viewpoint.

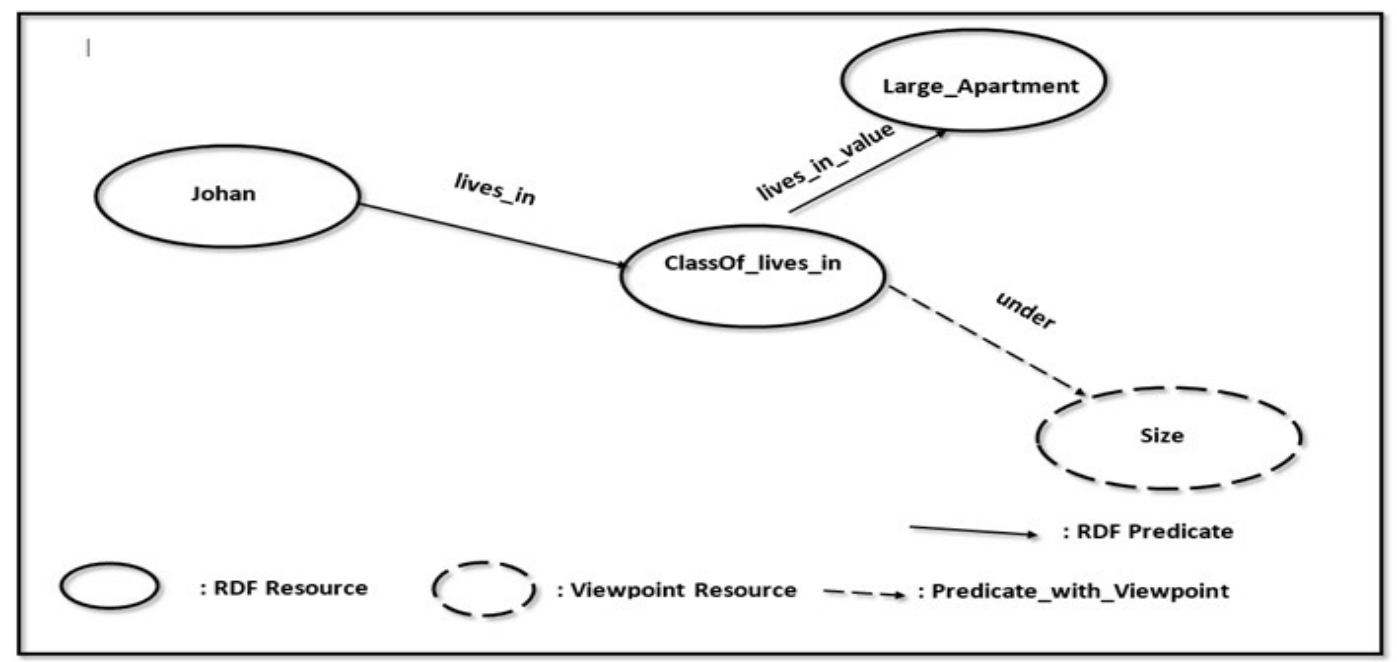

Fig. 4. RDF graph representation of an example of Case 5: a resource that is described by another resource. This description is defined according to a given viewpoint.

\section{QUERYING OF VP-RDF DESCRIPTIONS}

SPARQL (Protocol and RDF Query Language) is a query language that allows querying the RDF data [11] via SPARQL queries. Each query represents a set of triplet patterns [11]. The subject, the predicate and the object of the SPARQL triplets can be variables [11].

In this section, we present an example of VP-RDF data querying using the SPARQL language.

We take the example of the VP-RDF document of the real estate domain. This document comports the list of descriptions of resources, by taking into the consideration the viewpoints, in real estate domain, where Case 1 (in the previous section) represents an extract of this document. Below, we present an example (as demonstration) of the SPARQL query of this extract.

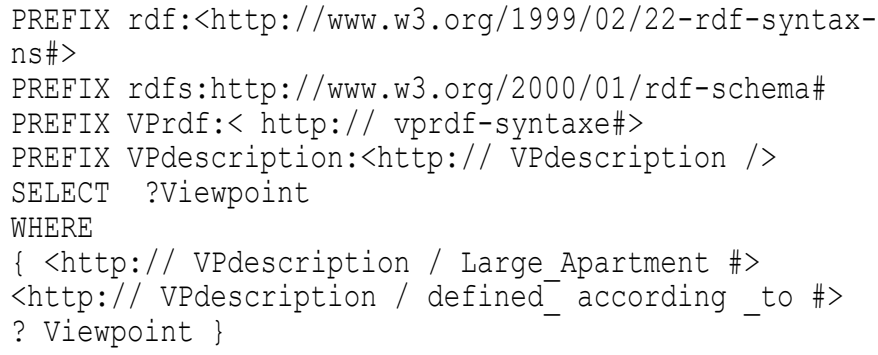

This query allows finding a viewpoint, where a given RDF resource is defined. The result of this query is the value "Size".

\section{Remarks:}

- http:// vprdf-syntaxe represents the URI of the VP-RDF elements proposed in Section 3.

- http:// VPdescription / represents the URI of the VP-RDF document save location.

\section{RESULTS AND DISCUSSION}

This section comprises four subsections. The first one allows showing the place of our work in the literature. In the second subsection, we prove that the set of the VP-RDF elements is satisfied and they are consistent and not redundant with the set of RDF elements. We present the goal of the choice to extend RDF in the third subsection. In the last subsection, we represent the performance of the proposed VP-RDF.

\section{A. Place of the VP-RDF in the Literature}

In this subsection, we show the place of our work in the literature. Therefore, we compare our work with the existing studies.

Several approaches have exploited RDF without extension to describe the resources. In [41], [42], the authors have presented metadata in RDF. Ashkpour et al. [43] have proposed a specific approach and model for creating an interlinked census dataset on the Semantic Web using the RDF. Sen et al. [44] are interested in the integration of meta-knowledge on the Web of data using RDF. In [45], the authors propose an algorithm of semantic relation recognition for natural language query over 
RDF data. Fan et al. [46] have proposed a fuzzy RDF(S) storage schema with fuzzy HBase databases (FHDBs). These approaches have not taken the notion of viewpoint into consideration.

Trichet, Aimé and Thovex in [47] presented a platform. In this platform, each user can use one or more ontologies to generate their annotation according to their own viewpoint. Then, a user represents annotation in RDF triplet. This platform allows taking multiple viewpoints and multiple users into consideration, but the notion of the viewpoint cannot appear clearly in the RDF representation.

In our work, the element viewpoint, Predicate_with_Viewpoint and VP-RDF statement allow representing clearly the notion of the viewpoint.

Other works have proposed extending RDF with contexts (e.g., in [19]-[24]).

In these works, the goal is to represent that an assertion is true only under a certain set of conditions (contexts). However, our goal is to represent explicitly the relation between a resource and a viewpoint and the relation between a predicate and a viewpoint. Moreover, the notion of the viewpoint is different from the notion of the context.

On the other hand, in order to represent the context, some works (e.g., [21] and [22]) have extended RDF by adding a new component to the RDF triple. In this case, the RDF context statement becomes quadruplet. The existing RDF query tools cannot query a quadruplet structure. Therefore, some authors have also proposed an extension of the SPARQL (e.g., [19]).

However, VP-RDF, that we propose, keeps the triplet structure like RDF. Therefore, the query languages (e.g., SPARQL) can query it.

\section{B. Proving of the Satisfiability, Consistency and Nonredundancy of the VP-RDF Elements}

VP-RDF is considered an extension of RDF by adding new elements. Therefore, it is necessary to prove the satisfiability of the new elements and to demonstrate that these elements do not generate inconsistency and redundancy.

In order to prove the satisfiability of an element, it is necessary to find that the set of instances of this element is different from the empty set $(\varnothing)$.

$C_{\text {viewpoint }}, C_{\text {predicate_with_viewpoint }}$ and $C_{\text {statement }}$ are the sets of instances of the elements VPrdf:Viewpoint, VPrdf:Predicate_with_Viewpoint, and VPrdf:Statement, respectively. We have seen in the use cases some examples of instances of the elements: VPrdf:Viewpoint, VPrdf:Predicate_with_Viewpoint and VPrdf:Statement. For example, in the real estate domain:

$$
\begin{array}{r}
C_{\text {viewpoint }}=\{\text { Size, Finance, Localisation }, \ldots\} \Rightarrow C_{\text {viewpoint }} \neq \varphi ; \\
C_{\text {predicate_with_viewpoint }}=\{\text { defined_according_to, under, } \ldots\} \\
\Rightarrow C_{\text {predicate_with_viewpoint }} \neq \varphi ;
\end{array}
$$

$$
\begin{aligned}
C_{\text {statement }}=\{(<\text { Large_Apartment }>,<\text { defined_in }> & ,<\text { Size }>), \ldots\} \\
& \Rightarrow C_{\text {statement }} \neq \varphi .
\end{aligned}
$$

Thus, we have proven the satisfiability of the new elements.

$C_{r d f s: R e s o u r c e}, C_{r d f: P r o p e r t y}$ and $C_{r d f: S t a t e m e n t}$ are the sets of instances of the elements rdfs:Resource, rdf:Property and rdf:Statement, respectively.

The elements VPrdf:Viewpoint, VPrdf:Predicate_with_Viewpoint, and VPrdf:Statement are the subclasses of rdfs:Resource, rdf:Property and rdf:Statement, respectively.

$$
\left(C_{\text {viewpoint }} \subset C_{\text {rds:Resource }}\right) \leftrightarrow\left(\forall i, i \in C_{\text {viewpoint }}\right.
$$

$$
\left.\rightarrow i \in C_{\text {rdfs:Resource }}\right) \text {. }
$$

Each viewpoint is an RDF resource, but not all RDF resources are viewpoints. Thus, there are RDF resources that are not viewpoints. The site of the viewpoints represents a specification of the set of the RDF resources. Therefore, the element vprdf:viewpoint does not generate inconsistency (contradiction) or redundancy.

$$
\begin{aligned}
&\left(C_{\text {predicate_with_viewpoint }} \subset C_{\text {rdd:Property }}\right) \leftrightarrow \\
&\left(\forall i, i \in C_{\text {predicate_with_viewpoint }} \rightarrow i \in C_{\text {rdf:Property }}\right) .
\end{aligned}
$$

Every predicate_with_viewpoint is an RDF property that allows linking a resource to a viewpoint (the viewpoint should be only an object of the property predicate_with_viewpoint). Not all predicates are predicate_with_viewpoint. Therefore, the element vprdf:predicate_with_viewpoint does not generate inconsistency or redundancy.

$$
\begin{aligned}
& \left(C_{\text {statement }} \subset C_{\text {rdf:Statement }}\right) \leftrightarrow \\
& \quad\left(\forall i, i \in C_{\text {statement }} \rightarrow i \in C_{\text {rdf:Statement }}\right) .
\end{aligned}
$$

VP-RDF statement keeps the triplet structure of RDF statements (subject, predicate, object). The VP-RDF subject is an RDF resource that is different from viewpoints. The VPRDF predicate is an instance of vprdf:predicate_with_viewpoint. The VP-RDF object is an instance of vprdf:viewpoint. Therefore, the element vprdf:statment does not generate inconsistency or redundancy.

We have proven that VP-RDF is an extension of RDF by adding new elements, which do not generate inconsistency and redundancy.

\section{Proving the Choice of the RDF}

We will explain the choice of RDF. Hemam and Boufaida [15] proposed MVP-OWL as an extension of OWL to represent the notion of viewpoint within the ontology. Existing tools for the manipulation of ontologies cannot exploit the MVP-OWL elements.

The query languages (e.g., SPARQL) can query VP-RDF data. This shows the reason to start introducing the viewpoint on the low level (i.e., RDF). 
OWL is a language that represents the ontologies. The ontology allows describing the semantics of a given domain. The ontologies can be used to describe the semantic of resources via semantic annotation techniques. However, semantic annotations of resources on the web are represented in either RDF or XML.

All typical cases of a large linked dataset are available in RDF (e.g., DBPedia that makes the content of Wikipedia).

On the other hand, users do not need to rely on complex technologies (e.g., OWL); they simply describe resources into RDF using its vocabulary [48]. Making use of property hierarchies to regroup properties and using higher concept properties and classes when selecting specific information are very complex tasks for users. Therefore, users avoid using description based on the capabilities of RDFS and OWL. These reasons have made us propose extending RDF.

$\mathrm{RDF}$ represents the foundation for other technologies, including OWL. Therefore, if we can introduce the viewpoint in RDF, it will be easy to exploit the viewpoint in OWL, OWL2, RDFa, etc. Since RDFs and OWL are based on RDF elements, they will be based on VP-RDF elements as well. Thus, the capabilities of RDFS and OWL will be augmented.

\section{Performance of the $V P-R D F$}

VP-RDF, which we propose, allows representing explicitly the relation between a resource and a viewpoint and the relation between a property and a viewpoint. This explicitly representation facilitates the user to express the viewpoint without complication or confusion.

SPARQL does not support any reasoning mechanism. The user will program the reasoning at the generation of the requests (i.e., the user must form a "smart" request). Therefore, the user must know the structure of the RDF graph of the document. However, a majority of users do not know the structure of the RDF graph of the web resources (e.g., the users of the Wikipedia do not know the structure of the DBPedia). Thus, SPARQL cannot find the viewpoint in the RDF documents because it does not support any reasoning mechanism. However, SPARQL does not need reasoning on VP-RDF document to find the viewpoint because VP-RDF allows representing explicitly the viewpoint.

The user can mention the viewpoint in the request on the VPRDF documents. Then, SPARQL can select the responses that are linked directly to this viewpoint because VP-RDF allows representing explicitly the relations between the viewpoint and resources and the relations between predicates and the viewpoint. Intelligent systems on the web can detect the user's viewpoint from their requests via the several techniques, such as machine learning. Then, SPARQL can select the responses from the VP-RDF documents that are relevant to the user's interests. Therefore, VP-RDF helps intelligent systems serve the user.

Let us refer to the example of the real estate site; a user wants to know all the existing properties of an apartment that describe its size.

In VP-RDF, the properties (e.g., height, area, the number of rooms, etc.) are linked directly to the viewpoint size. Thus,
SPARQL provides the user with only properties of this apartment that are linked to the viewpoint size (i.e., height, area, the number of rooms, etc.). SPARQL does not provide the user with the properties: price, rent, location, etc. Therefore, the responses are relevant to the user's interests.

However, in the RDF data querying, SPARQL provides the user with all the properties of this apartment because RDF cannot show that such properties are linked to the viewpoint size.

Thus, the search with VP-RDF is limited only to the properties of this apartment that are linked to the viewpoint size. Response time of the VP-RDF data querying can be optimised.

\section{CONCLUSION}

In this paper, we have proposed VP-RDF as an extension of RDF by adding new elements that allow introducing explicitly the viewpoint in the description of the resources and their relationships. These new elements are satisfied and they do not generate inconsistency and redundancy with the RDF elements.

The VP-RDF statement that allows linking a resource to a viewpoint keeps the triplet structure. This structure allows the query languages (e.g., SPARQL) to query efficaciously the VPRDF graphs.

VP-RDF allows representing explicitly the relation between a resource and a viewpoint and the relation between a property and a viewpoint. This characteristic allows the user to easily create their description of resources by taking into consideration the viewpoint. The benefit of the integration of the viewpoint in $\mathrm{RDF}$ is to enable SPARQL to provide the user with responses according to their interests.

VP-RDF helps intelligent systems provide the user with relevant responses after the detection of their interests.

The VP-RDF is useful in several tasks (e.g., the multiviewpoint semantic annotation [37], [38]).

RDF represents a foundation for other technologies of semantic web. Semantic web technologies will help various domains resolve their problems [49]. On the other hand, RDF is also the basic structure of linked data [50]. Therefore, VPRDF will be a foundation to introduce the viewpoint in the other technologies (e.g., OWL2, RDFa, etc.).

Sen et al. [44] have assumed that the integration of metaknowledge on the web of data is essential to support trustworthiness. Meta-knowledge describes how the data are generated, manipulated, and disseminated [44]. Therefore, as future work, we plan to introduce the notion of the viewpoint in the construction of meta-knowledge on the web of data.

\section{REFERENCES}

[1] D. Heckmann, Ubiquitous User Modeling. Berlin: Aka, 2005

[2] O. Djama and Z. Boufaida, "Instantiation of the Multi-Viewpoints Ontology from a Resource," International Journal of Computers and Applications, pp. 1-12, Jan. 2020. https://doi.org/10.1080/1206212X.2020.1711615

[3] F. Al-akashi, "SAMA: A Real-Time Web Search Architecture," International Journal of Computers and Applications, pp. 1-8, Dec. 2020. https://doi.org/10.1080/1206212X.2020.1859245

[4] T. Berners-Lee, J. Hendler and O. Lassila, "The Semantic Web," Journal of Scientific American, vol. 284, no. 5, pp. 34-43, May 2001. https://doi.org/10.1038/scientificamerican0501-34 
[5] G. Klyne and J. Carroll. (2004, Feb.). Resource Description Framework (RDF): Concepts and Abstract Syntax [Online]. Available: http://www.w3.org/TR/2004/REC-rdf-concepts-20040210/

[6] O. Lassila and R. R. Swick. (1998, Feb.). Resource Description Framework (RDF) Model and Syntax Specification [Online]. Available: https://www.w3.org/TR/1998/WD-rdf-syntax-19980819/

[7] I. Herman, "W3C Semantic Web Frequently Asked Questions," Nov. 2009. [Online]. Available: http://www.w3.org/2001/sw/SW-FAQ

[8] T. Berners-Lee, J. Hollenbach, L. Kanghao, J. Presbrey, E. Prud'hommeaux and M. C. Schraefel, "Tabulator Redux: Browsing and Writing Linked Data," presented at the 1st Workshop on Linked Data on the Web (LDOW2008), Beijing, China, 2008.

[9] C. Bizer, T. Heath and T. Berners-Lee, "Linked Data: The Story so Far," International Journal on Semantic Web and Information Systems, vol. 5 , no. 3, pp. 1-22, 2009. https://doi.org/10.4018/jswis.2009081901

[10] A. Polleres, A. Hogan, R. Delbru and J. Umbrich, "RDFS and OWL Reasoning for Linked Data," in Reasoning Web, in Semantic Technologies for Intelligent Data Access, S. Rudolph, G. Gottlob, I. Horrocks and F. Harmelen, Eds. Berlin (Heidelberg): Springer-Verlag, 2013, pp. 91-149. https://doi.org/10.1007/978-3-642-39784-4_2

[11] D. Beckett and J. Broekstra. (2013, Mar.). SPARQL Query Results XML Format [Online]. Available: http://www.w3.org/TR/2013/REC-rdfsparql-XMLres-20130321

[12] M. Minsky, "A Framework for Representing Knowledge," in The Psychology of Computer Vision, P. H. Winston, Ed. New York: McGrawHill, 1975, pp. 211-217

[13] O. Marino, "Raisonnement classificatoire dans une représentation à objets multi-points de vue," PhD thesis, University of Joseph Fourier, Grenoble, France, 1993

[14] T. L. Bach, "Construction d'un Web sémantique multi-points de vue," $\mathrm{PhD}$ thesis, Mines ParisTech (école des Mines de Paris), Sophia Antipolis, France, 2006.

[15] M. Hemam and Z. Boufaida, "MVP-OWL: A Multi-Viewpoints Ontology Language for the Semantic Web," International Journal of Reasoningbased Intelligent Systems, vol. 3, no. 3/4, pp. 147-155, Nov. 2011. https://doi.org/10.1504/IJRIS.2011.043539

[16] R. Dieng, O. Corby, F. Grandon, A. Giboin, J. Golebiowska, N. Matta and M. Ribière, Méthodes et outils pour la gestion des connaissances: une approche pluridisciplinaire du knowledge management, 2nd ed. Paris, France: Dunod, 2005.

[17] G. Falquet and C.L. Mottaz, "Navigation hypertexte dans une ontologie multi-points de vue," presented at the conference NîmesTIC'2001, Nîmes, France, 2001

[18] K. Khan, A. Ullah and B. Baharudin, "Pattern and Semantic Analysis to Improve Unsupervised Techniques for Opinion Target Identification," Kuwait Journal of Science, vol. 43, no. 1, pp. 129-149, Feb. 2016.

[19] A. Analyti, C. V. Damasio and I. Pachoulakis, "Nested Contextualised Views in the Web of Data," International Journal of Web Engineering and Technology, vol. 10, no. 1, pp. 31-64, May. 2015. https://doi.org/10.1504/IJWET.2015.069360

[20] H. Cherfi, O. Corby, C. Faron-Zucker, K. Khelif and M. T.Nguyen, "Semantic Annotation of Texts with RDF Graph Contexts," presented at Conceptual Structures: Knowledge Visualization and Reasoning. International Conference on Conceptual Structures (ICCS'08), CEURWS, Toulouse, France, 2008.

[21] L. Hollink, G. Schreiber, J. Wielemaker and B. Wielinga, "Semantic Annotation of Image Collections," presented at Workshop on Knowledge Markup and Semantic Annotation (KCAP'03), Florida, USA, 2003

[22] O. Khriyenko and V. Terziyan, "A Framework for Context-Sensitive Metadata Description," International Journal of Metadata, Semantics and Ontologies, vol. 1, no. 2, pp. $154-164$, Oct. 2006. https://doi.org/10.1504/IJMSO.2006.011011

[23] H. Stoermer, P. Bouquet, I. Palmisano and D. Redavid, "A Context-Based Architecture for RDF Knowledge Bases: Approach, Implementation and Preliminary Results," in Web Reasoning and Rule Systems, M. Marchiori, J. Z. Pan and C. S. Marie, Eds. Berlin (Heidelberg): Springer, 2007, pp. 209-218. https://doi.org/10.1007/978-3-540-72982-2 15

[24] R. Vanlande and C. Nicolle, "Context DataModel Framework: Semantic Facilities Management," International Journal of Product Lifecycle Management, vol. 3, no. 2/3, pp. 165-177, Nov. 2008 https://doi.org/10.1504/IJPLM.2008.021440
[25] A. Martin, S. Emmenegger, K. Hinkelmann and B. Thonssen, "A Viewpoint-Based Case-Based Reasoning Approach Utilising an Enterprise Architecture Ontology for Experience Management," Enterprise Information Systems, vol. 11, no. 4, pp. 551-575, 2017. https://doi.org/10.1080/17517575.2016.1161239

[26] J. Kingston, "Multi-Perspective Ontologies: Resolving Common Ontology Development Problems," Expert Systems with Applications, vol. 34, no. 1, pp. 541-550, Jan. 2008. https://doi.org/10.1016/i.eswa.2006.09.040

[27] M. Hemam, M. Djezzar and T. Djouad, "A Description Logics Based Approach for Building Multi-Viewpoints Ontologies," International Journal of Computer and Information Engineering, vol. 10, no. 12, pp. 2111-2116, 2016. https://doi.org/10.5281/zenodo.1339734

[28] E. Zemmouri, H. Behja, A. Marzak and B. Trousse, "Ontology-Based Knowledge Model for Multi-View KDD Process," International Journal of Mobile Computing and Multimedia Communications, vol. 4, no. 3, pp. 21-33, 2014. https://doi.org/10.4018/jmcmc.2012070102

[29] M. Hemam, M. Djezzar and A. S. Zianou, "Multi-Viewpoints Ontological Knowledge Representation: A Fuzzy Description Logics Based Approach," presented at the Fourth International Conference on Engineering \& MIS 2018 (ICEMIS'18), Istanbul, Turkey, 2018. https://doi.org/10.1145/3234698.3234761

[30] M. Zhitomirsky-Geffet, E. S. Erez and B. I. Judit, "Toward Multiviewpoint Ontology Construction by Collaboration of Nonéexperts and Crowdsourcing: The Case of the Effect of Diet on Health," Journal of the Association for Information Science and Technology, vol. 68, no.3, pp. 681-694, Mar. 2017. https://doi.org/10.1002/asi.23686

[31] M. Oprea, "A Case Study of Collaborative Ontology Development for Higher Education," International Journal of Artificial Intelligence (IJAI), vol. 14, no. 2, pp. 70-97, Oct. 2016.

[32] S. Gorshkov, S. Kralin and M. Miroshnichenko, "Multi-Viewpoint Ontologies for Decision-Making Support," in Knowledge Engineering and Semantic Web. KESW 2016. Communications in Computer and Information Science, A. C. Ngonga Ngomo and P. Kremen, Eds. Suisse (Switzerland): Springer-Cham, 2016, pp. 3-17. https://doi.org/10.1007/978-3-319-45880-9 1

[33] M. Hemam, M. Djezzar and Z. Boufaida, "Multi-Viewpoint Ontological Representation of Composite Concepts: A Description Logics-Based Approach," International Journal of Intelligent Information and Database Systems, vol. 10, no.1/2, pp. 51-68, Aug. 2017. https://doi.org/10.1504/IJIIDS.2017.086193

[34] M. Hemam, "An Extension of the Ontology Web Language with MultiViewpoints and Probabilistic Reasoning," International Journal of Advanced Intelligence Paradigms, vol. 10, no. 3, pp. 247-265, Jan. 2018. https://doi.org/10.1504/IJAIP.2018.090789

[35] M. Djezzar and Z. Boufaida, "Ontological Classification of Individuals: A Multi-Viewpoints Approach," International Journal of Reasoningbased Intelligent Systems, vol. 7, no. 3/4, pp. 276-285, Nov. 2015. https://doi.org/10.1504/IJRIS.2015.072954

[36] L. Djakhdjakha, M. Hemam and Z. Boufaida, "Towards a Representation for Multi-Viewpoints Ontology Alignments," International Journal of Metadata, Semantics and Ontologies, vol. 9, no. 2, pp. 91-102, Apr. 2014. https://doi.org/10.1504/IJMSO.2014.060324

[37] O. Djama and Z. Boufaida, "Multi-Viewpoints Semantic Annotation of XML Documents," in Proceedings of the World Congress on Engineering 2013, International Association of Engineers: 2013, S. I. Ao, L. Gelman, D. W. L. Hukins, A. Hunter and A. M. Korsunsky, Eds. London (U.K): Newswood Limited, 2013, pp. 390-394.

[38] O. Djama, "Annotation sémantique Multi-Points de Vue (MPV) de ressources et leur exploitation à travers un langage basé $\mathrm{RDF}, " \mathrm{PhD}$ thesis, University of Abdelhamid Mehri-Constantine 2, Constantine, Algeria, 2020.

[39] P. F. Patel-Schneider, "Building the Semantic Web Tower from RDF Straw," presented at the 19th International Joint Conference on Artificial Intelligence (IJCAI 2005), Scotland, Edinburgh, 2005.

[40] Y. Gryaznov and P. Rusakov, "Analysis of RDF Syntaxes for Semantic Web Development," Applied Computer Systems, vol.18, no. 1, pp. 33-42, 2015. https://doi.org/ doi:10.1515/acss-2015-0017

[41] H. Cui, K. Jiang and P. P. Sanyal, "From Text to RDF Triple Store: An Application for Biodiversity Literature," Journal of the American Society for Information Science and Technology, vol. 47, no. 1, pp. 1-2, Feb. 2011. https://doi.org/10.1002/meet.14504701415 
[42] B. Martins and M. J. Silva, "The WebCAT Framework Automatic Generation of Meta-Data for Web Resources," presented at the 2005 IEEE/WIC/ACM International Conference on Web Intelligence (WI 2005), Compiegne, France, 2005.

[43] A. Ashkpour, A. Merono-Penuela and K. Mandemakers, "The Aggregate Dutch Historical Censuses," Historical Methods: A Journal of Quantitative and Interdisciplinary History, vol. 48, no. 4, pp. 230-245, Oct. 2015. https://doi.org/10.1080/01615440.2015.1026009

[44] S. Sen, M. C. Malta, B. Dutta and A. Animesh-Dutta, "State-of-the-Art Approaches for Meta-Knowledge Assertion in the Web of Data," IETE Technical Review, Sep. 2020. https://doi.org/10.1080/02564602.2020.1819891

[45] X. Hu, J. Duan and D. Dang, "Crowdsourcing-Based Semantic Relation Recognition for Natural Language Questions over RDF Data," Enterprise Information Systems, vol. 13, no. 7-8, pp. 935-958, Mar. 2019. https://doi.org/10.1080/17517575.2019.1597385

[46] T. Fan, L. Yan and Z. Ma, "Storing and Querying Fuzzy RDF(S) in HBase Databases," International Journal of Intelligent Systems, vol. 35, no. 4, pp. 751-780, Jan. 2020. https://doi.org/10.1002/int.22224

[47] F. Trichet, X. Aimé and C. Thovex, "OSIRIS: Ontology-Based System for Semantic Information Retrieval and Indexation Dedicated to Community and Open Web Spaces," in Hershey Handbook of Research in Culturally-Aware Information Technology: Perspectives and Models, E. G. Blanchard and D. Allard, Eds. PA: Information Science Publishing, 2010, pp. 465-483. https://doi.org/10.4018/978-1-61520-883-8.ch021
[48] B. Menon, "Comprendre les standards du web de données," I2D Information, données \& documents, vol. 53, no. 2, pp. 32-34, 2016. https://doi.org/10.3917/i2d.162.0032

[49] A. Patel and S. Jain, "Present and Future of Semantic Web Technologies: A Research Statement," International Journal of Computers and Applications, Jan. 2019. https://doi.org/10.1080/1206212X.2019.1570666

[50] A. Billey, R. Rendall and K. Wesley, "Foundations in Linked Data for Serialists," The Serials Librarian. vol. 76, no. 1-4, pp. 16-29, May. 2019. https://doi.org/10.1080/0361526X.2019.1598748

Ouahiba Djama was born in Constantine, Algeria. She received her Engineer degree in Computer Science in 2005 and M. sc. in 2010, both from University of Mentouri, Constantine, Algeria. She obtained a Doctoral degree in Computer Science in 2020 from the University of Abdelhamid Mehri Constantine 2, Ali Mendjeli, Constantine, Algeria.

She is currently an Assistant Professor at the University of Mentouri BrothersConstantine 1, Constantine, Algeria. She is also an Attached Member of the SI\&BC research group at Lire Laboratory of the University of Abdelhamid Mehri-Constantine 2, Constantine, Algeria. Her research interests include knowledge representation and reasoning, formal knowledge representation for semantic web, ontology development, web technologies, big data, bioinformatics, artificial intelligence and computer applications.

E-mail: djama ouahiba@umc.edu.dz 